\title{
RMT-HBase: A Distributed Framework for Trajectory Storage and Query
}

\author{
Cheng Yang, Jiamin Lu, Jun Feng +, Dingsheng Li \\ College of Computer and Information, Hohai University, Nanjing, China
}

\begin{abstract}
The rapid development of science and technology has resulted in smartphones, global positioning systems and location-based services becoming immensely popular. Massive amounts of trajectory data from mobile objects have been applied in many domains. Many related trajectory storage methods based on HBase focus on design of Rowkey to adapt to different scenarios, don't fully use the unique index META table of HBase. In this paper, we propose a distributed framework RMT-HBase to support efficient trajectory storage and query processing. We redesign both the upper and lower index structure of HBase to optimize the storage of trajectory data. To improve the query efficiency of spatial-temporal data, we design three kinds of trajectory query algorithms. We compare the spatial-temporal data query performance of RMT-HBase with the RMHBase on synthetic datasets, and experimental results show that the RMT-HBase outperforms the RM-HBase for the same query.
\end{abstract}

Keywords: Spatial-temporal index, query algorithm, road network.

\section{Introduction}

The rapid development of technologies such as the Global Positioning System (GPS), Radio Frequency Identification (RFID) and location-based services (LBSs) has made it possible to gather massive trajectory data, which contains a large amount of hidden and meaningful information and can be well applied in many domains. Hence, it's essential to study an efficient method to store large scale of trajectory data.

Some existing trajectory data storage methods are designed based on conventional spatial index, which utilized the advantages of simple operations and fast retrieval, but not consider the temporal dimension adequately. In addition, these methods can't adapt to continuous change of data load, any update of index may result in huge time consumption. To fix these issues, some researchers propose trajectory storage methods based on cloud platforms. With their storage and coordinator component, realizing large scale of trajectory data storage and real-time data processing efficiently. However, the calculation layer of Hadoop consumes much system resource inevitably.

A lot of distributed databases have been applied to store huge volume data, such as HBase. However, it's lack of an efficient index for managing spatial-temporal data. To fix this problem, Nishimura et al. [1] proposed MD-HBase, which store the coding range of consecutive subspace in META table. MD-HBase improves the performance of spatial-temporal query, but it only creates upper index without consider the lower index, which limits the query performance. Chen et al. [2] proposed STEHIX which creates s-index and t-index to retrieve spatial and temporal attributes respectively, but the maintenance of index is costly. In addition, STEHIX doesn't consider redesigning the Rowkey of HBase to optimize the index and improve the query performance.

Both MD-HBase and STEHIX divide the space into equal size, which is not suitable for road network, because the mobile objects in road network is not distributed equally and result in dead-space problem, that is, some storage space never be accessed. To fix this problem, Feng et al. [3] proposed a mobile objects spatial-

\footnotetext{
+ Corresponding author.

E-mail address: fengjun@hhu.edu.cn
} 
temporal index framework in road network RM-HBase, which creates spatial index RN-tree to store road segments into leaf nodes, which solves the dead-space problem efficiently. However, RM-HBase creates tindex instead of considering temporal dimension of META table, it's costly to maintain multi-level secondary index except the main index. Also, its redesign of Rowkey is not suitable for high-efficiency query of trajectory data.

Motivated by these problems, we propose a distributed framework RMT-HBase to support efficient trajectory storage and query processing. We redesign the META table of HBase, considering both spatial and temporal dimensions to quickly retrieve the trajectory data, which also simplifies the index structure. We redesign the Rowkey of HBase to optimize the retrieval speed of the trajectory data. To improve the query efficiency of spatial-temporal data, we design three kinds of trajectory query algorithms, an object-time-based trajectory query algorithm, a space-time-based trajectory query algorithm, and a time-based trajectory query algorithm. The main contribution of this paper is three fold:

- We redesign both the upper and lower index structure of HBase to improve the retrieval speed of the trajectory data.

- To improve the query efficiency of spatial-temporal data, we design three kinds of trajectory query algorithms.

- We compare the spatial-temporal data query performance of RMT-HBase with the RM-HBase on synthetic datasets, and experimental results show that the RMT-HBase outperforms the RM-HBase for the same query.

The rest of this paper is organized as follows. Section II reviews the related work of trajectory storage methods. We present the storage design of RMT-HBase in Section III. In Section IV, we present three kinds of trajectory query algorithms. We present the experimental results in Section V and Section VI concludes the paper.

\section{Related Work}

In this section, we first review previous trajectory storage methods, then we review some spatial-temporal index based on HBase.

\subsection{Trajectory Storage Methods}

Some previous trajectory storage methods make improvement based on classic spatial index structures:

[4][5][6][7] proposed spatial index based on tree structure index. Philippe et al. [4] proposed TrajStore, maintains an adaptive grid scheme that partitions space into a number of grid cells, co-locates and compresses spatially and temporally adjacent segments on disk and dynamically adapts the index as new data is loaded or the query workload evolves.

[8]proposed spatial index based on inverted index. To solve efficient and effective KNN Sequence search with approximate n-grams, Wang et al. [8] implemented a two-level inverted index. In the upper-level of index, $\mathrm{n}$-grams that are derived from the sequence database are stored in an inverted file with their references to the original sequences. In the lower-level of index, each distinct n-gram from the upper-level is further decomposed into smaller sub-units, and inverted lists are constructed to store the references to the upper-level grams for each sub-unit.

[9][10] proposed spatial index based on grid index. Zheng et al. [9] develops a novel hybrid grid index, GAT, to organize the trajectory segments and activities hierarchically, which enables pruning the search space by location proximity and activity containment simultaneously and process the queries efficiently. Unfortunately, these methods may result in data redundancy. In addition, the space partition granularity directly influences the storage usage, which is not suitable for large scale of spatial data analysis.

Some trajectory storage methods are combined with cloud platforms:

[11][12] combine cloud platforms such as Hadoop and Spark for storage and query of large scale trajectory data. Eldawy et al. [11] proposed SpatialHadoop, a full-fledged MapReduce framework with native support for spatial data. In the storage layer, SpatialHadoop adapts traditional spatial index structures, Grid, R-tree and $\mathrm{R}+$-tree, to form a two-level spatial index. Since input files in Hadoop are non-indexed heap files, the 
performance is limited as the input has to be scanned. To overcome this limitation, SpatialHadoop employs spatial index structures within Hadoop Distributed File System (HDFS) as a means of efficient retrieval of spatial data. These methods utilize advantages of cloud platforms, but calculation with MapReduce consumes much system resource inevitably.

\subsection{Spatial-temporal Index Based on HBase}

[13][14] use Geohash as RowKey to design spatial index. To solve the nearby vehicle query issue in intelligent transportation field, Shen et al. [13] builds an HBase spatial index as RowKey, which maps two dimensional traffic data of spatial location information into one-dimensional Geohash encoding by dichotomy. The Rowkey is composed of Geohash encoding and entity ID. Zhang et al. [15] proposed HBaseSpatial to resolve the problems of storage of big vector spatial data. They create an index table, redesign the RowKey, which composed of the longitude, latitude, ids of each vector data. When doing range query, HBaseSpatial first finds correspond field in the index table, and then find all the vector data records in the data table according to the field. Chen et al. [16] proposed STEHIX which adapted to two-level architecture of HBase, makes it suitable for processing spatial-temporal queries. STEHIX is composed of index in the meta table and region index for indexing inner structure of HBase regions. Zhang et al. [17] redesign the Rowkey to index the indoor moving object trajectory, which composed of Object, Time and Cell. Given a mobile object and time period, the semantic trajectory can be queried. Nishimura et al. [1] proposed MD-HBase, which leverages a multidimensional index structure layered over a key-value Store and demonstrates how the K-d tree and the Quad tree can be layered over a range partitioned key-value store to provide scalable multi-dimensional data infrastructure. Feng et al. [3] proposed RM-HBase, which creates spatial index RN-tree to store road segments into leaf nodes, solves the dead-space problem efficiently. These spatial-temporal methods based on HBase utilize the RowKey to adapt different scenarios, however, most of them didn't fully use the unique index META table of HBase to efficiently combined spatial with temporal dimension, which limits the retrieval speed of trajectory data.

\section{RMT-HBase}

\subsection{Overview}

The RMT-HBase contains two parts: spatial-temporal data storage and trajectory query. The spatialtemporal data storage redesigns both the upper and lower structure of HBase. The trajectory query consists of three trajectory query algorithms: the object-time-based trajectory query algorithm, space-time-based trajectory query algorithm, and time-based trajectory query algorithm. The system architecture of RMT-HBase is shown in Fig. 1.

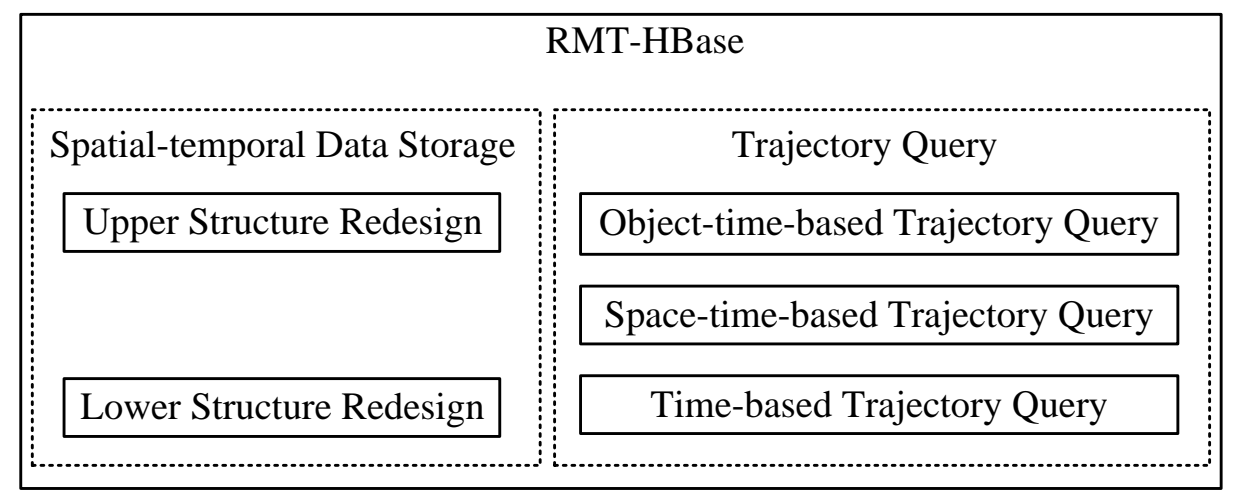

Fig. 1: System architecture of RMT-HBase.

In the spatial-temporal data storage, we redesign both the upper and lower structure of HBase. We propose a two-level index structure by fully using the unique index META table, considers both spatial and temporal dimensions, and we redesign the Rowkey of HBase to optimize the storage of spatial-temporal data.

In the trajectory query, we propose three trajectory query algorithms: the object-time-based trajectory query algorithm, space-time-based trajectory query algorithm, and time-based trajectory query algorithm to improve the efficiency of trajectory query. 


\subsection{Upper Structure Redesign}

We redesign META table with a two-level index, consider the difference between the spatial and temporal dimension. We divide the META table into two parts: space segments SS and time segments TS, the space segments are the first-level index (spatial index) and time segments are the second-level index(temporal index).

We use RN-tree [3] as spatial index to store mobile object data, which solves dead space problem caused by equal division of space. The structure of RN-tree is shown in Fig. 2.
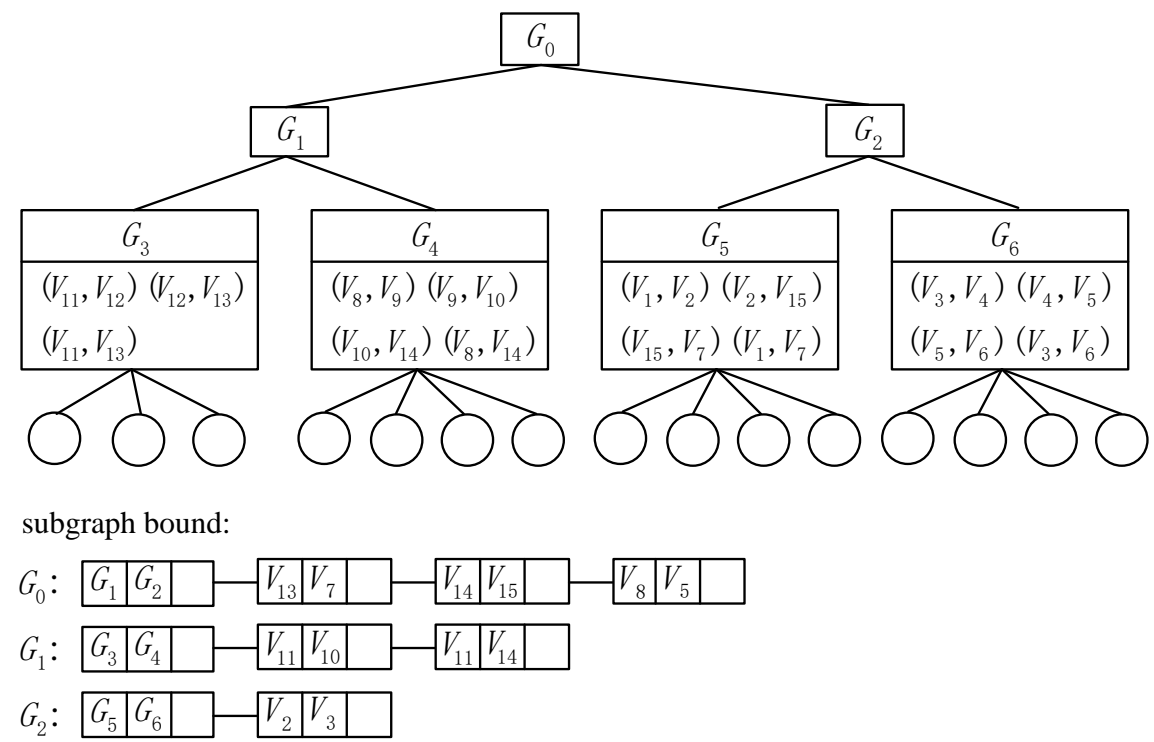

Fig. 2: The structure of RN-tree index.

$\mathrm{RN}$-tree is a spatial index in road network. It borrows the main idea of G-tree, solves the dead-space problem caused by equal space partition. RN-tree first divides the cover range of $G_{0}$ into two sub spaces: $G_{1}$ and $G_{2}$, then divides the sub spaces continuously according to the road segments, finally put all road segments into leaf nodes.

The leaf nodes store the start and end points, space range of the road segments. The non-leaf nodes store the cover bound of its child graphs. RN-tree is a multi-path balancing tree. Each leaf node points to one row of META table, which stores the mapping relations between road segment and Region and HRegionServer. When user makes a space query, the system first uses RN-tree to find the set of road segments, and then locates the Region and HRegionServer of mobile objects of the road segment with META table.

As the temporal attribute is incremental, that is difficult to be maintained. Also, it can't be treated as the prefix of RowKey, which may cause the data distribution hotspot problem. Hence, we design the temporal index as the second-level index. The index structure of META table is shown in Fig. 3.

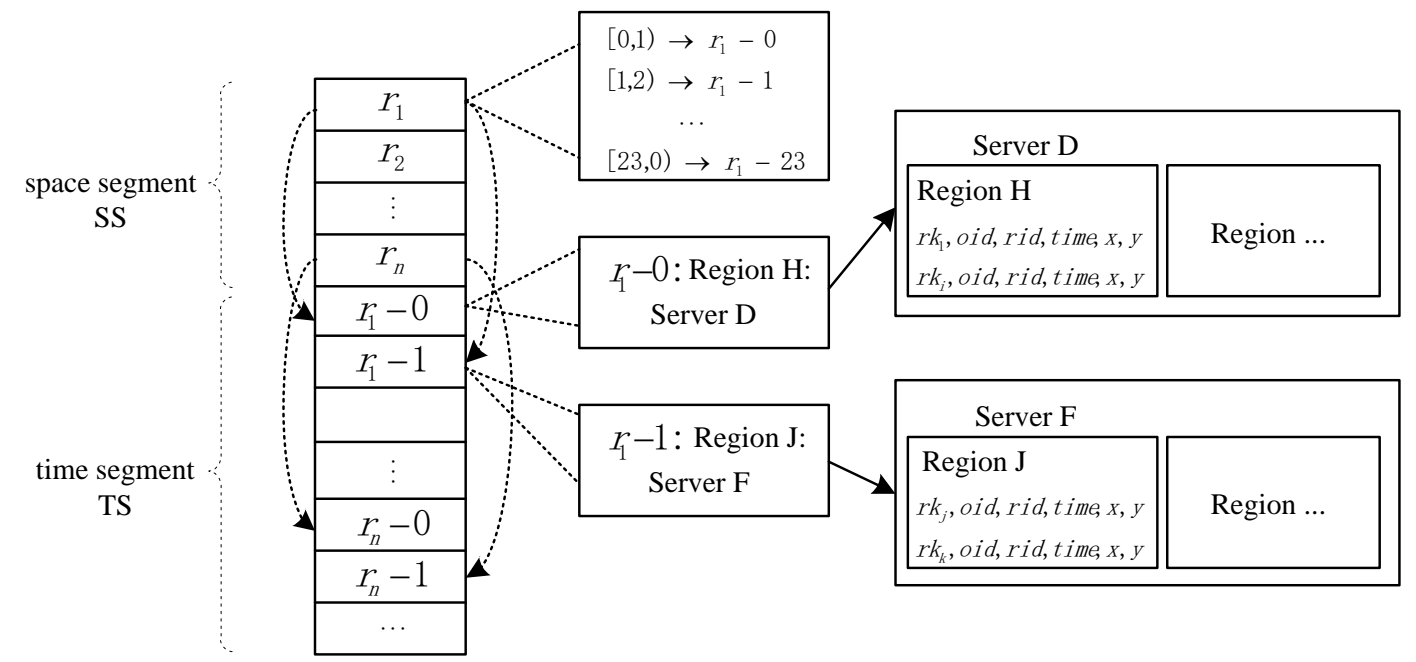

Fig. 3: Index structure of META table. 
As shown in Fig. 3, the structure of the META table is divided into two parts: a space segment and a time segment. The space segment SS stores all the identifier of road segments, that is, each entry corresponds to a road segment in the road network. The structure of each entry is $<$ road_id, $t_{s-}$ list $>$, where road_id is the id of a road segment, and the elements in the $t_{s-}$ list point to the entry in TS.

The time period is stored in TS. Each entry in the space segment SS corresponds to a time period in TS. The time period is 24 hours, and the granularity of the time segment in the region is one hour. Any value of time mode 24 can be matched to the corresponding time segment. The structure of entry in the time segments TS is $<$ time_id,region_id $>$, where time_id is the time segment identifier, and region_id represents the corresponding region identifier of the time segment.

As shown in Fig. 3, the META table structure contains two parts: time segments and space segments. The space segment SS consists of $\mathrm{n}$ road segments $r_{1}, r_{2}, \ldots, r_{n}$. In the time segment TS, the time period is set to 24 hours, the time granularity was set to one hour. For example, when the moving object is located in the road segment $r_{1}$ and the time mode 24 is 0 , then, the moving object data are stored in the Region $\mathrm{H}$ of the HRegionServer $\mathrm{D}$ pointed to by the $\mathrm{r}_{1}-0$ identifier.

\subsection{Lower Structure Redesign}

The lower structure of the RMT-HBase redesigns the RowKey according to the characteristics of the trajectory data, and retains the object index structure o-index of the RM-HBase[3]. The structure of the o-index is shown in Fig. 4.

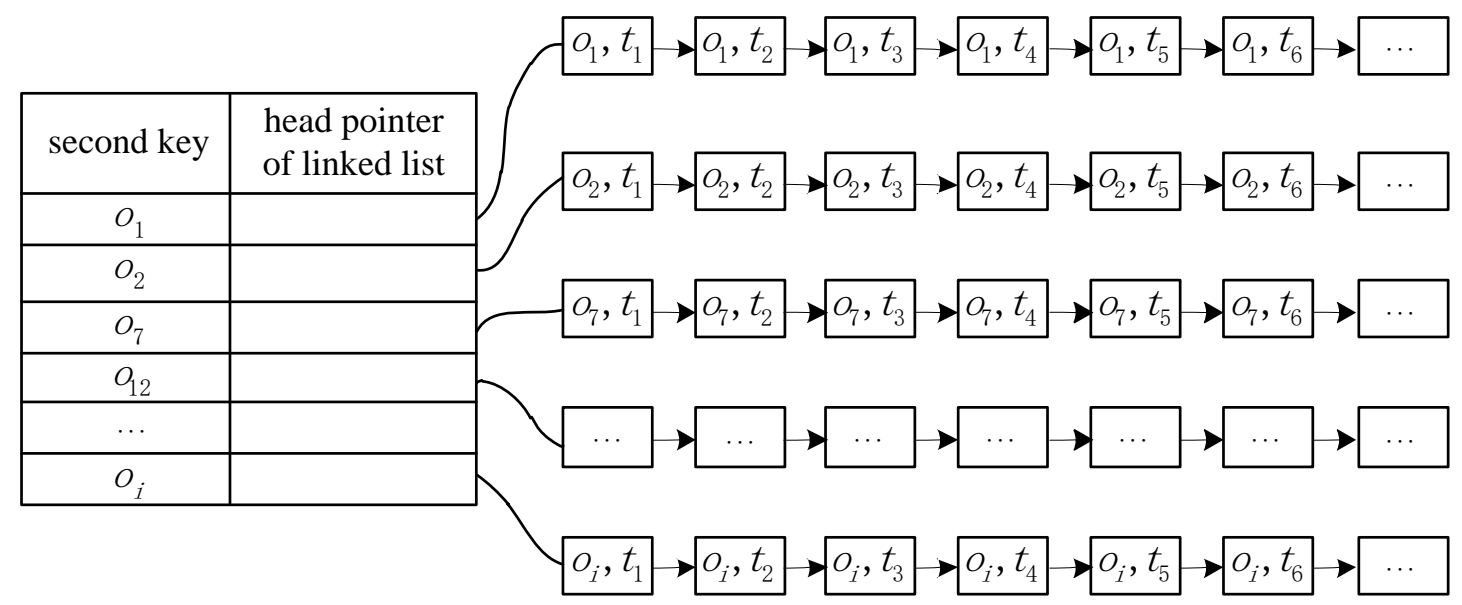

Fig. 4: The structure of o-index.

We use o-index as the secondary index while the META table is the main index. The table on the left stores the mobile object identifier as the second key and its head pointer. The linked list on the right stores the location of the moving object data corresponding to $o_{i}$, and sorted by the record time $t_{i}$ of the moving object. When querying the mobile object, we first query the left table to ensure the object is stored in current block, and then search all records with the head pointer, else skip this block. The o-index can efficiently improves the query efficiency.

Du et al. [18] and Le et al. [19] improved the spatial-temporal retrieval efficiency of the moving object by redesigning the RowKey. Based on the characteristics of the RowKey lexical order storage and the sequence characteristics of the moving object, we redesign the RowKey such that the moving object data can be stored in the order of time sequences, that is, the data are stored in trajectory format, as expressed by Formula. 1.

$$
\text { RowKey }=\text { oid }+T+\text { rid }
$$

The RowKey contains three attributes: the moving object identifier oid, the data entry time $\mathrm{T}$, and the road segment identifier rid. This design allows the data to be first stored according to the oid, next stored according to $\mathrm{T}$, then stored in according to rid. Because the trajectory data with time sequences features, redesigning the RowKey has the effect of ensuring that the order in which the moving object data are stored coincides with the order of the trajectory sequence, improving the efficiency of trajectory query. 


\section{Trajectory Query}

We propose three trajectory query algorithms: the object-time-based trajectory query algorithm, spacetime-based trajectory query algorithm, and time-based trajectory query algorithm to improve the efficiency of trajectory query.

\subsection{Object-time-based Trajectory Query}

For object-time-based trajectory query, it finds trajectory data set of specific moving object in a given time period.

Definition 1 (object-time-based trajectory query) Given an object identifier oid and a time period $\left[t_{s}, t_{e}\right]$, the object-time-based trajectory query returns the mobile object trajectory data set.

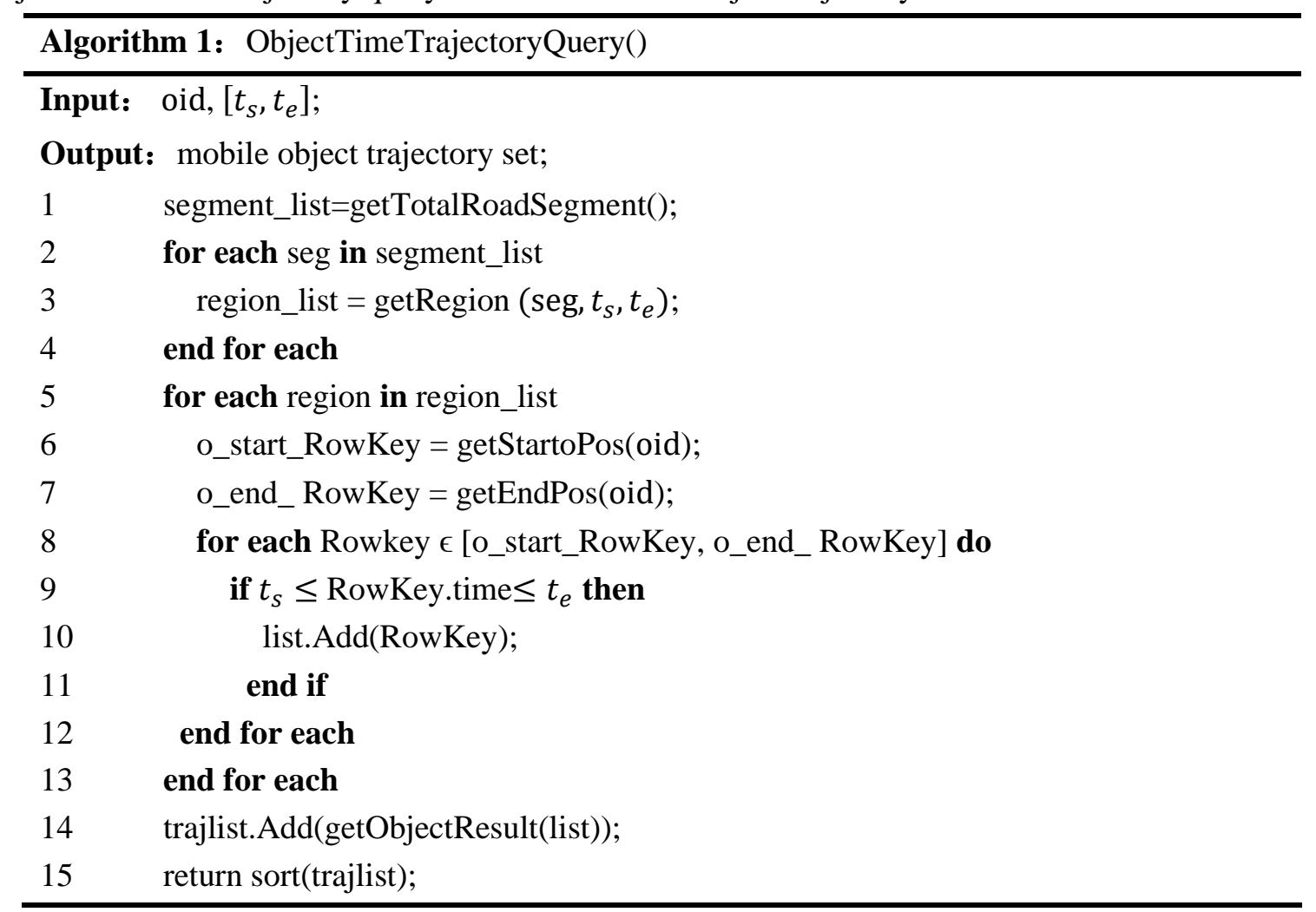

The object-time-based trajectory query algorithm is outlined in Algorithm 1. For an input mobile object identifier oid and time period $\left[\mathrm{t}_{\mathrm{s}}, \mathrm{t}_{\mathrm{e}}\right]$, we first load all road segments into segment_list (line 1), and find corresponding Regions and HRegionServers of the given time period with META table (line 3). We get the start and end Rowkey of input object with o-index. If a Region has no object, then skip this Region (line 5-7). We put Rowkey which satisfy the given time period $\left[\mathrm{t}_{\mathrm{s}}, \mathrm{t}_{\mathrm{e}}\right]$ into list (line 8-12). Finally, we add the mobile object trajectory data into trajlist and returned (line 14-15).

\subsection{Space-time-based Trajectory Query}

For space-time-based trajectory query, it finds trajectory data set of all moving objects in a given space range and time period.

Definition 2 (space-time-based trajectory query) Given a space range $\left(\left[x_{1}, x_{2}\right],\left[y_{1}, y_{2}\right]\right)$ and a time period $\left[t_{s}, t_{e}\right]$, the space-time-based trajectory query returns the trajectory data set of all mobile objects.

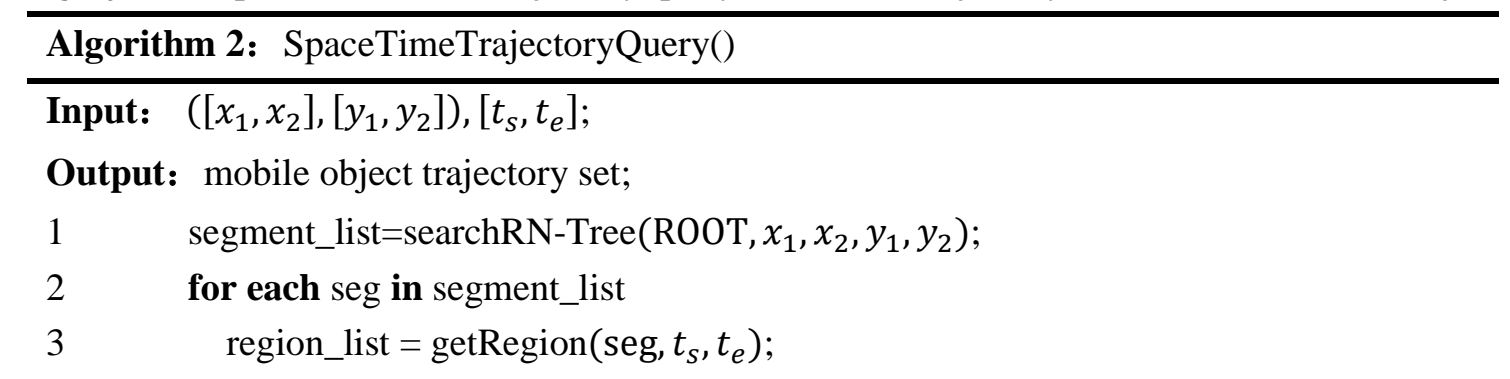




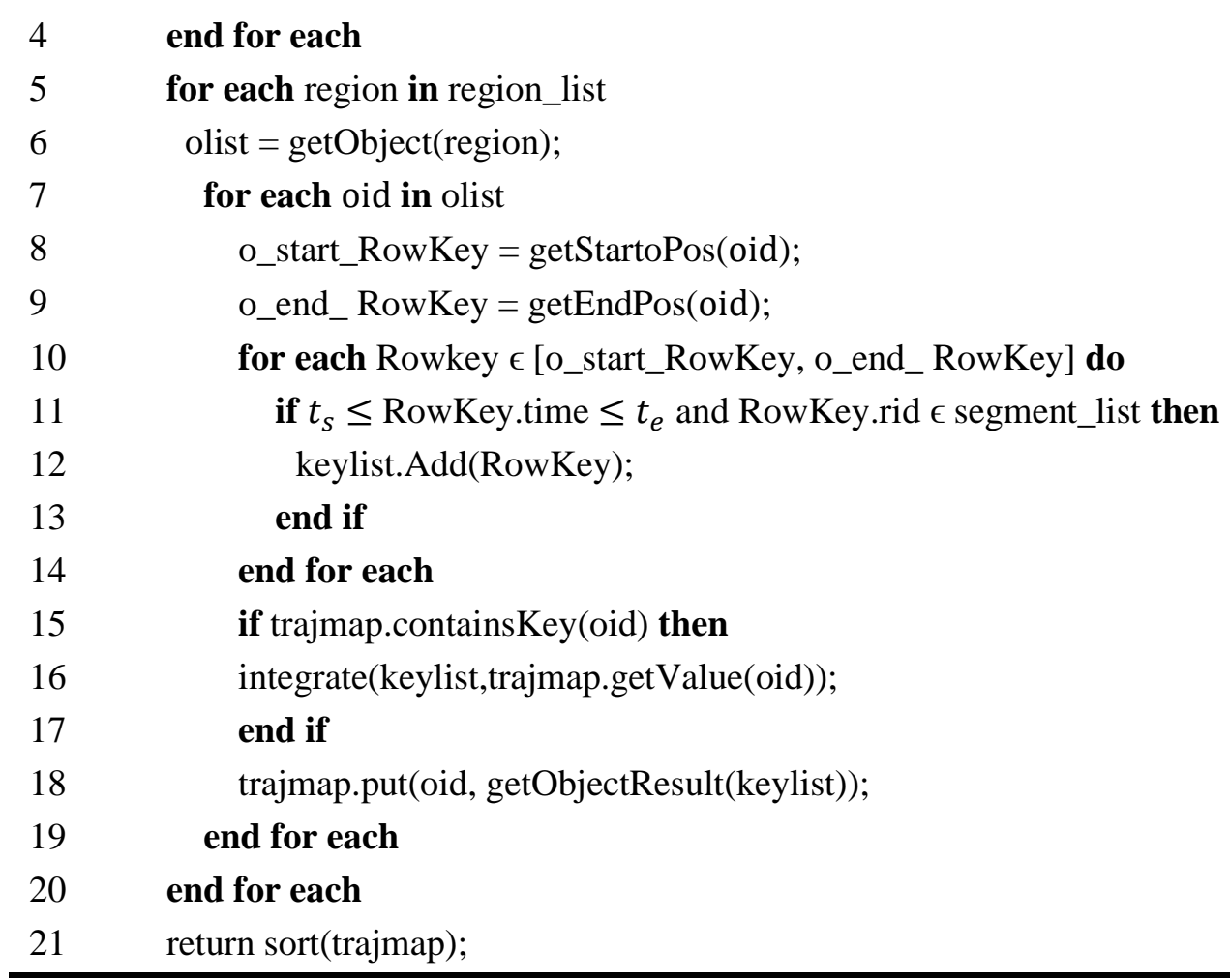

The space-time-based trajectory query algorithm is outlined in Algorithm 2. For an input space range $\left(\left[x_{1}, x_{2}\right],\left[y_{1}, y_{2}\right]\right)$ and time period $\left[t_{s}, t_{e}\right]$, we first find all the road segments in given space range with RNtree and put them into segment_list (line 1). ROOT is the root of RN-tree. We find corresponding Regions and HRegionServers of the given time period with META table (line 3). We get mobile objects' oid in the Region with o-index and put them into olist (line 6). We get the start and end Rowkey of mobile objects. If a Region has no object, then skip this Region (line 7-9). We put Rowkey which satisfy the given time period $\left[t_{s}, t_{e}\right]$ and space range into keylist. We add each mobile object and its trajectories into trajmap (line 15-18). Finally, the mobile object trajectory data set is returned (line 21).

\subsection{Time-based Trajectory Query}

For time-based trajectory query, it finds trajectory data set of all moving objects in a given time period.

Definition 3 (time-based trajectory query) Given a time period $\left[t_{s}, t_{e}\right]$, the time-based trajectory query returns the trajectory data set of all mobile objects.

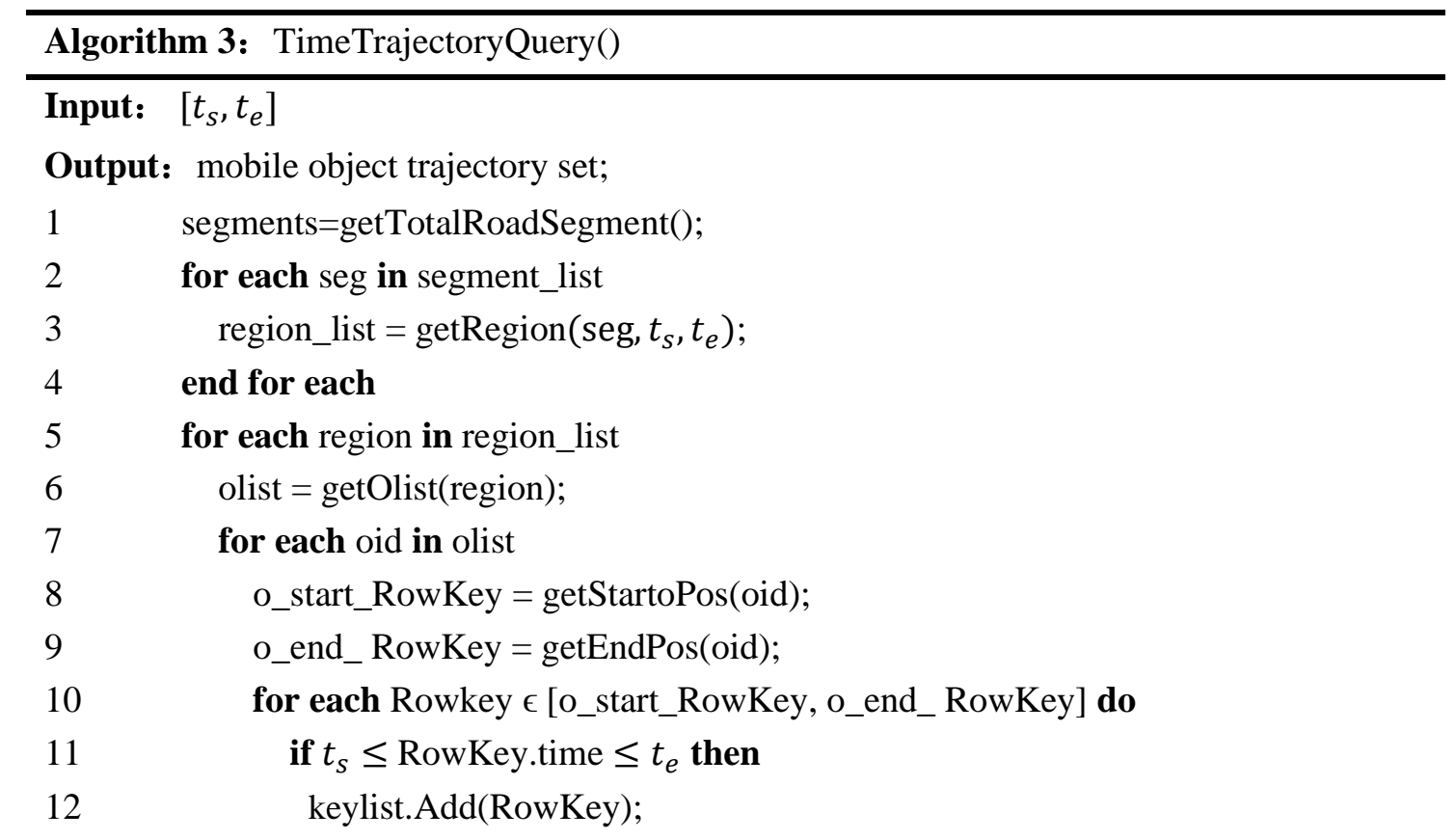


end if

\section{end for each}

if trajmap.containsKey(oid) then

integrate(keylist,trajmap.getValue(oid));

end if

trajmap.put(oid, getObjectResult(keylist));

\section{end for each}

end for each

return sort(trajmap);

The time-based trajectory query algorithm is outlined in Algorithm 3. For an input time period $\left[t_{s}, t_{e}\right]$, we first load all road segments into segment_list (line 1), and find corresponding Regions and HRegionServers of the given time period with META table (line 3). We get mobile objects in the Region with o-index and put them into olist (line 6). We get the start and end Rowkey of all mobile objects with o-index. If a Region has no object, then skip this Region (line 7-9). We put Rowkey which satisfies the given time period $\left[t_{s}, t_{e}\right]$ and add them into keylist (line 10-12). We add each mobile object and its trajectories into trajmap (line 15-18). Finally, the mobile object trajectory data set is returned (line 21).

\section{Experiments}

\subsection{Experimental Setup}

We generate a synthetic trajectory dataset with road network data generator Thomas Brinkhoff [20], which simulates trajectory of mobile object in Oldenburg city. The test area contains 830,000 road segments and trajectory data of 10,000 moving objects in a week. We replicate data to adapt to different requirements.

We use Hadoop-2.7.3 and HBase-1.2.5 to build a Hadoop cluster, with five nodes: one NameNode as HMaster, four DataNodes as HRegionServers. Each node is equipped with Intel Core i5-6500, 3.20GHz CPU, $8 \mathrm{G}$ memory and 500G disk, the operating system is Ubuntu14.04 and network bandwidth is $100 \mathrm{Mbps}$.

\subsection{Comparison of RMT-HBase and RM-HBase}

This part we evaluate three trajectory query algorithms and compare the different performance between RMT-HBase and RM-HBase[3].

For the object-time-based trajectory query, the amount of data generated by the moving object may affect the query performance. Therefore, we test the time consuming of query by varying the amount of data. The experimental results are shown in Fig 5(a), which indicates that the object-time-based trajectory query of RMTHBase is superior to that of RM-HBase. There are two reasons for the above phenomenon: First, the RMTHBase redesign the META table considering both spatial and temporal dimensions, thus, it can efficiently locate the corresponding HRegionServer of query time, whereas the RM-HBase needs to send a query request to all HRegionServers. Second, RMT-HBase redesigns the Rowkey, which makes mobile object data store in trajectory sequence order, further improves the trajectory efficiency of moving object.

For the space-time-based trajectory query, the factors that may affect the query performance are the query window size and the data size. Therefore, we first test the time consumed by the query while the query window size is varied, we set six different sizes of rectangular query windows, namely $1 * 1,2 * 2,3 * 3,4 * 4,5 * 5,6 * 6$. Then we test the time consumed by the query by varying the amount of data. Figure 5(b) shows the time consumption of the space-time-based trajectory query while the query window size is varied. The experimental results show that, as the size of the query window increases, the time consuming of RMT-HBase and RMHBase increases continuously, but the query efficiency of RMT-HBase is superior to that of RM-HBase. To test the impact of the data size on the query, the size of the query window is set to $6^{*} 6$, and the data size varies from 40 to $320 \mathrm{~GB}$. Figure 5(c) shows the time consuming of the space-time-based trajectory query while the amount of data generated by the moving objects gradually increases. The performance of RMT-HBase is better than that of RM-HBase. The reason for above phenomenon is that the design of the META table of the RMTHBase considers the time dimension, thereby enabling the query to efficiently locate the corresponding Region 
of the query time period. At the same time, the structure of the RowKey is improved in the Region, such that the moving object is stored in the order of the trajectory sequence, which further improves the efficiency of the trajectory query.

(a) Effect of data size on query per formance

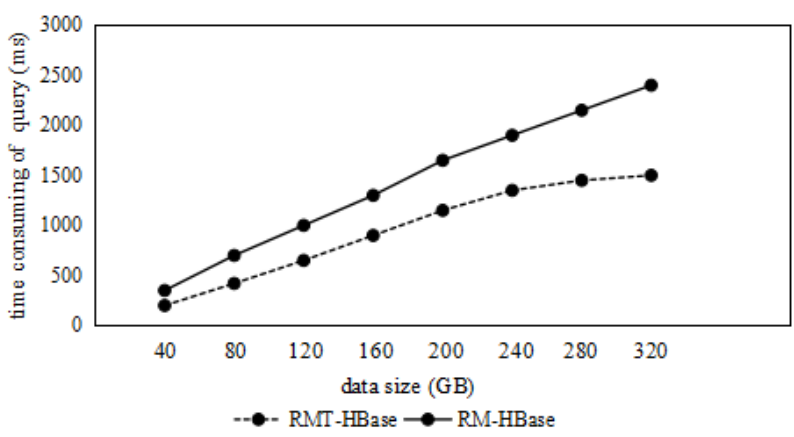

(c) Effect of data size on query per formance

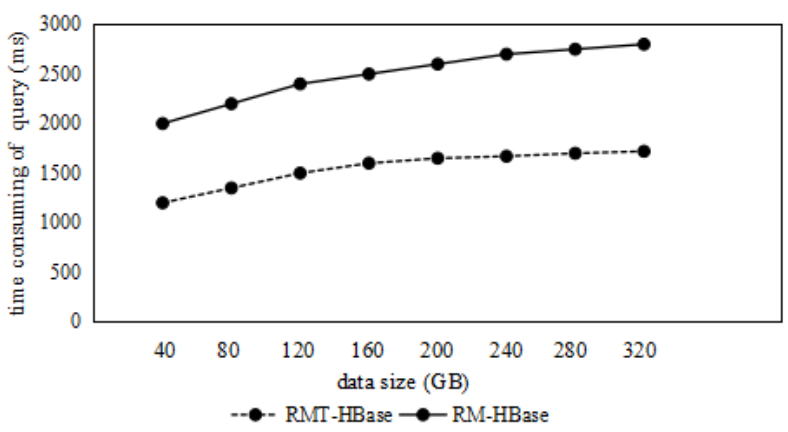

(b) Effect of query window on query performance

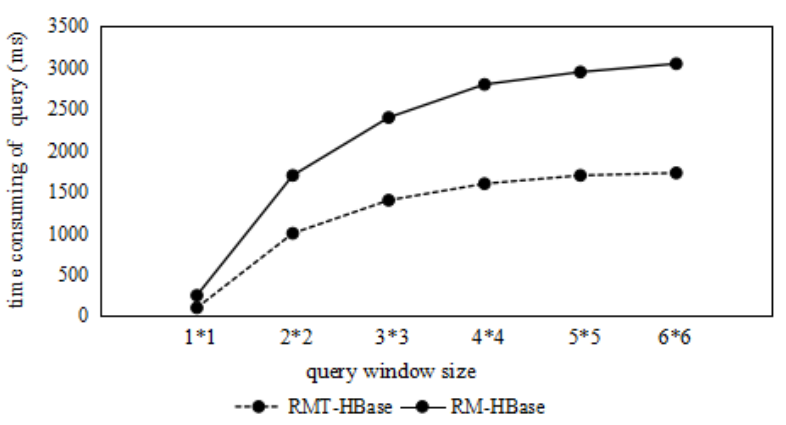

(d) Effect of data size on query performance

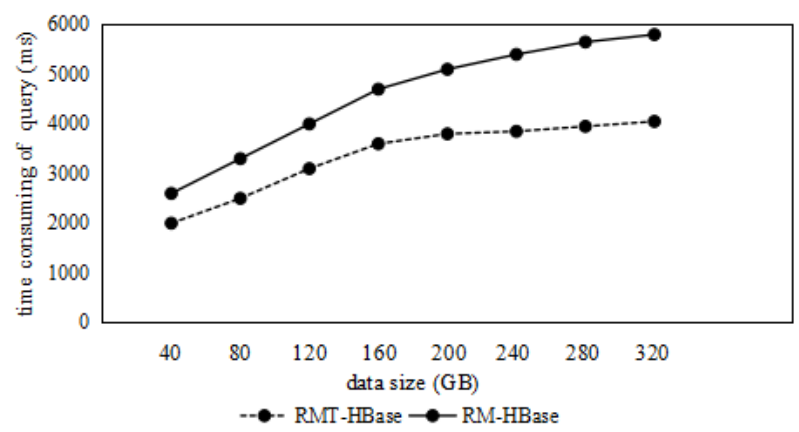

Fig. 5: Comparison of RMT-HBase and RM-HBase.

For the time-based trajectory query, the amount of data generated by the moving object may affect the query performance. Therefore, this experiment mainly tests the time consumed by the query while the amount of data changes. Figure 5(d) shows the time consumed by the time-based trajectory query while the amount of the moving object data gradually increases. The RMT-HBase outperforms RM-HBase in terms of time-based query performance. There are two reasons for this phenomenon: the improvement of the index structure of the upper level of RMT-HBase takes the temporal dimension into account whereas the RM-HBase only considered the spatial dimension. When the query is based on time period, all Regions of RM-HBase need to be queried, while the RMT-HBase only finds the corresponding Regions of query time period, thereby improving the performance of the time-based trajectory query. Second, the structure of the RowKey of RMT-HBase is redesigned, such that its data storage is in the order of the moving object trajectory sequence, which improves the efficiency of the trajectory query.

\section{Conclusion}

In this paper, we proposed RMT-HBase, a new distributed spatial-temporal index framework based on HBase. We redesign both the upper and lower index structure of HBase to optimize the storage of trajectory data. To improve the query efficiency of spatial-temporal data, we design three kinds of trajectory query algorithms. Extensive experimental results show that the RMT-HBase outperforms RM-HBase significantly.

\section{Acknowledgements}

This work was supported in part by the National Key R\&D Program of China under Grant 2018YFC0407901 and Grant 2017YFC0405806, in part by the Fundamental Research Funds for the Central Universities under Grant 2019B64614 and Grant 2019B15514, in part by the National Natural Science Foundation of China under Grant 61602149, Grant 61370091, and Grant 61602151, and in part by the Jiangsu Collaborative Innovation Center for Cultural Creativity under Grant XYN1702. 


\section{References}

[1] S. Nishimura, S. Das, D. Agrawal. MD-HBase: A Scalable Multi-Dimensional Data Infrastructure for Location Aware Services[C]// Proc. of the IEEE International Conference on Mobile Data Management. IEEE Computer Society. 2011:7-16.

[2] X. Chen, C. Zhang, B. Ge. Spatio-temporal queries in HBase[C]// Proc. of the IEEE International Conference on Big Data. IEEE, 2015:1929-1937.

[3] J. Feng, D. Li, J. Lu, L. Zhang. Spatio-temporal index method of moving object in road network based on HBase[J]. Journal of Computer Applications. 2018, 038(006):1575-1583,1590.

[4] P. Cudré-Mauroux, E. Wu, S R. Madden. TrajStore: An Adaptive Storage System for Very Large Trajectory Data Sets[C]// Proceedings of the 26th International Conference on Data Engineering. 2010:109-120.

[5] S. Ranu, P. Deepak, A D. Telang, et al. Indexing and matching trajectories under inconsistent sampling rates[C]// Proc. of the 31 st IEEE International Conference on Data Engineering. 2015:999-1010.

[6] K. Zheng, B. Zheng, J. Xu, et al. Popularity-aware spatial keyword search on activity trajectories[J]. World Wide Web, 2016, 20(4):1-25.

[7] K. Zheng, G. Trajcevski, X. Zhou, et al. Probabilistic range queries for Uncertain Trajectories on road networks[C]// Proc. of the International Conference on Extending Database Technology. ACM, 2011:975-986.

[8] X. Wang, X. Ding, A. Tung, et al. Efficient and effective KNN sequence search with approximate n-grams[J]. Proceedings of the VLDB Endowment, 2013, 7(1):1-12.

[9] K. Zheng, S. Shang, N J. Yuan, et al. Towards efficient search for activity trajectories[C]// Proc. of the 2013 IEEE 29th International Conference on Data Engineering. 2013:230-241.

[10] B. Zheng, N. Yuan, K. Zheng, et al. Approximate keyword search in semantic trajectory database[C]// Proc. of the 2015 IEEE 31st International Conference on Data Engineering. 2015:975-986.

[11] A. Eldawy, M F. Mokbel. SpatialHadoop: A MapReduce framework for spatial data[C]// Proc. of the 31st IEEE International Conference on Data Engineering. 2015:1352-1363.

[12] D. Xie, F. Li, B. Yao, et al. Simba: Efficient In-Memory Spatial Analytics[C]// Proc. of the 2016 International Conference on Management of Data. 2016:1071-1085.

[13] D. Shen, J. Fang, Y. Han. A Nearby Vehicle Search Algorithm Based on HBase Spatial Index[C]// Proc. of the 2015 12th Web Information System and Application Conference (WISA). 2015:71-74.

[14] Y. Wu, X. Tang. Technology of distributed spatial database based on HBase[J]. Journal of Jilin University (Science Edition), 2016(6).

[15] N. Zhang, G. Zheng, H. Chen, et al. HBaseSpatial: A Scalable Spatial Data Storage Based on HBase[C]// Proc. of the 2014 IEEE 13th International Conference on Trust, Security and Privacy in Computing and Communications (TrustCom). 2014:644-65.

[16] X. Chen, C. Zhang, B. Ge, et al. Spatio-temporal queries in HBase[C]// Proc. of the 2015 IEEE International Conference on Big Data (Big Data). 2015:1929-1937.

[17] D. Zhang, C. Xie, T. Pei. Semantic Cell Oriented Indoor Moving Objects Index based on HBase[J]. Journal of GeoInformation Science. 2017, 019(003):307-316.

[18] N. Du, J. Zhan, M. Zhao, et al. Spatio-Temporal Data Index Model of Moving Objects on Fixed Networks Using HBase[C]// Proc. of the IEEE International Conference on Computational Intelligence \& Communication Technology, 2015:247-251.

[19] L. Van, A. Takasu. A Scalable Spatio-temporal Data Storage for Intelligent Transportation Systems Based on HBase[C]// Proc. of the IEEE International Conference on Intelligent Transportation Systems. 2015, 18: 2733-2738.

[20] T. Brinkhoff. A Framework for Generating Network-Based Moving Objects[J]. GeoInformatica, 2002, 6(2):153180. 